\section{Just heat and. . .purify}

Elastin-like polypeptides (ELPs) are pentapeptide oligomers that aggregate when heated above a critical temperature that is determined by the specific sequence of amino acids in the monomer. Meyer and Chilkoti have made use of this phenomenon to develop a strategy for the temperature-dependent purification of ELP-tagged recombinant proteins (see p. 1112). Their new system provides an inexpensive and simple alternative to chromatography-based purification schemes.

\begin{abstract}
On page 1093, Lee and colleagues describe a useful tool for the mouse geneticist-a system for knocking out gene function with precise control over the developmental stage, cell type, and site of the affected tissue. The system uses the bacteriophage Cre-lox system; Cre recombinase specifically excises sequences placed between lox sequences. By placing Cre recombinase expression under control of cell type-specific and tetracycline-inducible control elements, the gene of interest can be deleted only in the desired cell types after tetracycline administration-a boon for studying the function of essential genes (see also p. 1063).
\end{abstract}

\section{Potentiating biocontrol}

Baculoviruses are potentially effective and safe agents for controlling crop pest infestation. However, the high cost of producing the virus has hindered commercialization. Hukuhara and colleagues now have made this strategy more economical by lowering the amount of baculovirus needed for crop application (see p. 1122). Their engineered transgenic rice expresses a proteinaceous enhancing factor from an armyworm virus. Armyworm larvae feeding on the rice had increased susceptibility to baculovirus infec-

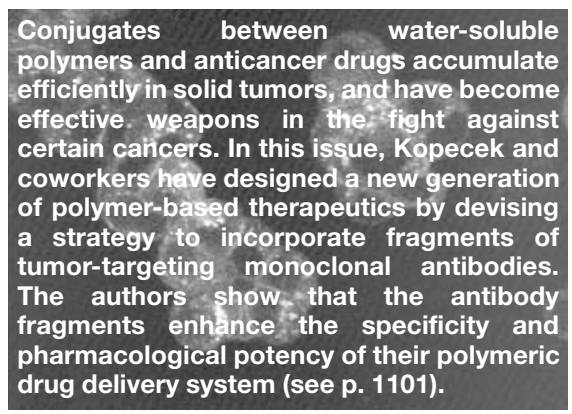

Research Briefs written by Natalie DeWitt and Robert Frederickson

The expression of recombinant proteins in the mammary glands of trangenic livestock has become an important strategy for the large-scale production of protein therapeutics. Despite the commercial success of this technology, other collectible body fluids are being investigated for recombinant protein production. In this issue, Dyck et al. demonstrate the feasibility of producing proteins in the male ejaculate by expressing human growth hormone in the seminal vesicles of transgenic mice (see p. 1087).

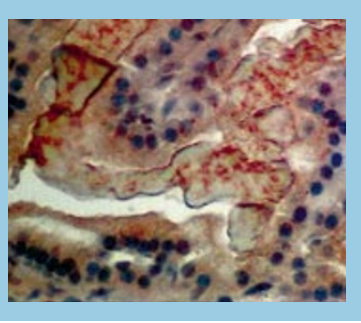

\section{Therapeutic proteins get a sugar coating}

Chinese hamster ovary $(\mathrm{CHO})$ cells are widely used to produce recombinant proteins as therapeutics for a variety of diseases. These proteins often contain carbohydrates that are critical for therapeutic efficacy. Now Krummen and colleagues have engineered $\mathrm{CHO}$ cells that overexpress two enzymes responsible for adding $\mathrm{N}$-linked oligosaccharides. Therapeutic IgGs produced in these cells had more complete carbohydrate modifications and persisted longer in an animal model system (see p. 1116).

There is a growing need for biocompatible materials suitable for the engineering of blood vessel replacements for the treatment of vascular disease. In this issue, Huynh et al. have fashioned scaffolds for such surgical replacements, derived from collagen biomaterial harvested from the submucosal, layer of the small intestine (see p. 1083). Within three months, the engineered grafts integrated into the host tissue, became recellularized by endogenous smooth muscle and endothelial cells, and were responsive to vasoactive factors:

\section{Antisense targets apoptosis}

Regulating the levels of antiapoptotic and preapoptotic proteins can sensitize cells to undergo apoptosis in response to chemotherapeutic agents. Using antisense oligonucleotides, Taylor et al. have altered splice-site selection in an endogenous apoptosis gene, $b c l-x$ (see p. 1097 and p. 1065). This treatment favors the production of a longer form of the mRNA, encoding for the proapoptotic protein $(\mathrm{Bcl}-\mathrm{xS})$, whereas it disfavors production of the antiapoptotic longer form (Bcl-xL) and sensitizes the cells to apoptotic stimuli.

\section{GPCR biosensor chip}

G protein-coupled receptors (GPCRs) are a large and ubiquitous class of membrane receptors that are important targets for drug discovery. Until now, most assays of GPCR function have been performed in whole cells or in transgenic animals, neither of which are amenable to high-throughput scale-up. In this issue, Vogel and coworkers have come up with an alternative platform by successfully immobilizing GPCRs on a chip surface and measuring their activation directly by surface plasmon resonance (SPR) (see p. 1105 and p. 1060).

\section{Honey, I shrank the cell sorter}

In this issue, Fu et al. describe a microfabricated fluorescence-activated cell sorter $(\mu \mathrm{FACS})$ that can sort micron-sized latex beads and bacterial cells (p. 1109 and p. 1061). The device is a silicone elastomer chip fabricated by soft lithography, with control over the fluidics systems that will allow cells to be sorted after observing them for a long time. The $\mu \mathrm{FACS}$ could provide a low cost, disposable alternative to conventional FACS, and has the potential for integration with other microfluidic systems on a chip.

A quick, non-
invasive tech-
nique for mon-
itoring the pres-
ence and ex-
pression of
transgenes in
crops could
benefit farmers
and lessen the
risk of transgene
escape. On page
1125, Stewart
and colleagues show that GFP is an
accurate marker of expression of the Bt
toxingene, providing a low-tech way to
detect transgenic plants in the field. They
determined the best GFP variant for this
purpose, and established that its
expression had no apparent fitness costs
to plants growing in the field.

